\title{
STUDIES IN FRACTIONAL POISSON MEASURES
}

\author{
JOSÉ LUÍS DA SILVA \\ Centro de Ciências Matemáticas, University of Madeira, Campus da Penteada \\ 9020-105 Funchal, Madeira, Portugal. \\ luis@uma.pt \\ MARIA JOÃO OLIVEIRA \\ Universidade Aberta, \\ Centro de Matemática e Aplicações Fundamentais, \\ Avenida Professor Gama Pinto, 2 \\ 1649-003 Lisbon, Portugal. \\ oliveira@cii.fc.ul.pt
}

\begin{abstract}
In this paper we investigate the quasi-invariance property of fractional Poisson measures with respect to the diffeomorphism subgroup and we construct spaces of test and generalized functions associated to the corresponding fractional Lebesgue-Poisson measures.

Keywords: Fractional Poisson measure; Diffeomorphism group; Test and generalized functions; Mittag-Leffler function.
\end{abstract}

PACS numbers: 11.30.Rd, 05.40.-a

\section{Introduction}

In the recent years the fractional Poisson process and its associated measure has become an object of study in finite dimensions, see for example Ref. 1, 2 and references therein. The corresponding infinite dimensional version of the fractional Poisson measure has been introduced in Ref. 3. In this paper we study further some properties of the latter measure, namely, the quasi-invariance property with respect to the diffeomorphism subgroup as well as the construction and description of a nuclear triple associated to the fractional Lebesgue-Poisson measure.

\section{Preliminaries}

In this section we briefly recall the concepts and results of fractional Poisson measures shown in Ref. 3. For this proposal, we start by fixing an underlying manifold $M$ with the Borel $\sigma$-algebra $\mathcal{B}(M)$ and a non-atomic Radon measure $\sigma$ which we assume to be non-degenerate (i.e., $\sigma(O)>0$ for all non-empty open sets $O \subset M$ ). We always assume that $M$ is a geodesically complete connected oriented (non-compact) 
Riemannian $C^{\infty}$-manifold. Having in mind the most interesting applications, we also assume that $\sigma(M)=\infty$. A typical example is $\mathbb{R}^{d}$ with $\sigma$ being the Lebesgue measure.

\subsection{Fractional Poisson measures}

On the space $\mathcal{D}:=\mathcal{D}(M)$ of $C^{\infty}$-functions with compact support in $M$ we consider the functional $C_{\alpha}$ given by

$$
C_{\alpha}(\varphi):=E_{\alpha}\left(\int_{M}\left(e^{i \varphi(x)}-1\right) d \sigma(x)\right), \quad \varphi \in \mathcal{D},
$$

where, for each $0<\alpha<1$ fixed, $E_{\alpha}$ is the Mittag-Leffler function of parameter $\alpha$,

$$
E_{\alpha}(z):=\sum_{n=0}^{\infty} \frac{z^{n}}{\Gamma(\alpha n+1)}, \quad z \in \mathbb{C} .
$$

For $\alpha=1$ we see that the Mittag-Leffler function coincides with the exponential function and thus (1) is the characteristic functional of the classic Poisson measure on the distribution space $\mathcal{D}^{\prime}:=\mathcal{D}^{\prime}(M)$ Ref. 4 . For $0<\alpha<1$ we have the following result cf. Ref. 3 .

Theorem 2.1. For each $0<\alpha<1$ fixed, the functional $C_{\alpha}$ in (1) is the characteristic functional of a probability measure on $\mathcal{D}^{\prime}$, denote by $\pi_{\sigma}^{\alpha}$ and called a fractional Poisson measure.

It is well known that the Mittag-Leffler function $E_{\alpha}$ is completely monotonic which is equivalent to the existence of a probability measure ${ }^{\mathrm{a}} \nu_{\alpha}$ on $\mathbb{R}_{0}^{+}:=[0, \infty)$ such that

$$
E_{\alpha}(-t)=\int_{0}^{\infty} e^{-t \tau} d \nu_{\alpha}(\tau), \quad \forall t>0 .
$$

The same results hold for any complex $z \in \mathbb{C}$ such that $\Re(z) \geq 0$, cf. Lemma 7 in Ref. 3. Using the representation (2) we may rewrite (1) as

$$
C_{\alpha}(\varphi)=\int_{0}^{\infty} \exp \left(\tau \int_{M}\left(e^{i \varphi(x)}-1\right) d \sigma(x)\right) d \nu_{\alpha}(\tau)
$$

showing that the integrand function corresponds to the characteristic function of the standard Poisson measure $\pi_{\tau \alpha}, \tau>0$. Therefore, by uniqueness, we have

$$
\pi_{\sigma}^{\alpha}=\int_{0}^{\infty} \pi_{\tau \sigma} d \nu_{\alpha}(\tau)
$$

which means that $\pi_{\sigma}^{\alpha}$ is the mixture of standard Poisson measures $\pi_{\tau \sigma}, \tau>0$. Since the standard Poisson measures are all supported on a subset of $\mathcal{D}^{\prime}$, see for

aThe measure $\nu_{\alpha}$ is absolutely continuous w.r.t. the Lebesgue measure and its density is related to the $\alpha$-stable probability distribution, see Ref. 5 for more details. 
example Ref. 4, property (3) then shows that $\pi_{\sigma}^{\alpha}$ has the same support, namely, the configuration space.

The configuration space $\Gamma:=\Gamma_{M}$ over the manifold $M$ is defined as the set of all locally finite subsets of $M$ (simple configurations),

$$
\Gamma:=\{\gamma \subset M:|\gamma \cap K|<\infty \text { for any compact } K \subset M\},
$$

where here (and below) $|A|$ denotes the cardinality of a set $A$. We identify each $\gamma \in \Gamma$ with a non-negative integer-valued Radon measure, $\Gamma \ni \gamma \mapsto \sum_{x \in \gamma} \delta_{x} \in \mathcal{M}(M)$, where $\delta_{x}$ is the Dirac measure with unit mass at $x, \sum_{x \in \emptyset} \delta_{x}:=0$ (zero measure), and $\mathcal{M}(M)$ denotes the set of all non-negative Radon measures on $\mathcal{B}(M)$. We may endow $\Gamma$ with the relative topology as a subset of $\mathcal{M}(M)$ with the vague topology. This means, the weakest topology on $\Gamma$ such that all mappings

$$
\Gamma \ni \gamma \mapsto\langle\gamma, f\rangle:=\int_{M} f(x) d \gamma(x)=\sum_{x \in \gamma} f(x)
$$

are continuous for any $f \in C_{0}(M)$ (the set of all continuous functions on $M$ with compact support). We denote the corresponding Borel $\sigma$-algebra on $\Gamma$ by $\mathcal{B}(\Gamma)$.

\subsection{Fractional Lebesgue-Poisson measures}

In order to introduce fractional Lebesgue-Poisson measures let us consider the space of finite configurations over $M, \Gamma_{0}$, defined as the disjoint union

$$
\Gamma_{0}:=\bigsqcup_{n=0}^{\infty} \Gamma_{M}^{(n)}
$$

of the spaces of $n$-point configurations $\Gamma_{M}^{(n)}:=\{\gamma \in \Gamma|| \gamma \cap M \mid=n\}, \Gamma_{M}^{(0)}:=$ $\{\emptyset\}$. One may endow $\Gamma_{0}$ with the disjoint union of the topological spaces $\Gamma_{M}^{(n)}$ and consider the corresponding Borel $\sigma$-algebra denoted by $\mathcal{B}\left(\Gamma_{0}\right)$. In fact, each $\Gamma_{M}^{(n)}$ has a topological structure introduced by the symmetric mapping $\operatorname{sym}_{M}^{n}\left(x_{1}, \ldots, x_{n}\right):=$ $\left\{x_{1}, \ldots, x_{n}\right\}$ from $\widetilde{M}^{n}$ (the set of all $\left(x_{1}, \ldots, x_{n}\right) \in M^{n}$ such that $x_{i} \neq x_{j}$ if $i \neq j$ ) to $\Gamma_{M}^{(n)}$. For more details see Ref. 6 .

For $\alpha=1$, the correlation measure corresponding to the Poisson measure $\pi_{\sigma}$ is the so-called Lebesgue-Poisson measure defined on $\mathcal{B}\left(\Gamma_{0}\right)$ by

$$
\lambda_{\sigma}^{1}:=\sum_{n=0}^{\infty} \frac{1}{n !} \sigma^{(n)}
$$

where $\sigma^{(n)}:=\sigma^{\otimes n} \circ\left(\operatorname{sym}_{M}^{n}\right)^{-1}$, see Ref. 6 for more details.

For $0<\alpha<1$ we have the following result, cf. Ref. 3 for more details.

Theorem 2.2. For each $0<\alpha<1$, the correlation measure corresponding to the fractional Poisson measure $\pi_{\sigma}^{\alpha}$ is the measure on $\left(\Gamma_{0}, \mathcal{B}\left(\Gamma_{0}\right)\right)$ given by

$$
\lambda_{\sigma}^{\alpha}:=\sum_{n=0}^{\infty} \frac{1}{\Gamma(\alpha n+1)} \sigma^{(n)}, \quad \sigma^{(n)}:=\sigma^{\otimes n} \circ\left(\operatorname{sym}_{M}^{n}\right)^{-1} .
$$


In other words, for

$$
E_{\alpha}^{(n)}(0)=\frac{n !}{\Gamma(\alpha n+1)}, \quad n \in \mathbb{N}_{0}
$$

denoting the $n$-th derivative of the Mittag-Leffler function $E_{\alpha}$ at the origin, one has $d \lambda_{\sigma}^{\alpha}=E_{\alpha}^{(|\cdot|)}(0) d \lambda_{\sigma}^{1}$. The measure $\lambda_{\sigma}^{\alpha}$ is called the fractional Lebesgue-Poisson measure.

\section{Quasi-invariance property of the fractional Poisson measure}

We denote by $\operatorname{Diff}(M)$ the group of all diffeomorphisms of $M$ and by $\operatorname{Diff}_{0}(M)$ the subgroup of all diffeomorphisms $\phi: M \longrightarrow M$ with compact support, i.e., $\phi(x)=x$ for all $x$ outside a compact set (depending on $\phi$ ). Any $\phi \in \operatorname{Diff}_{0}(M)$ defines a measurable mapping

$$
\Gamma \ni \gamma \mapsto \phi(\gamma):=\{\phi(x), x \in \gamma\} \in \Gamma .
$$

For any $\gamma \in \Gamma$, the configuration $\phi(\gamma)$ coincides with $\gamma$ for all but a finite number of points. The measurability of this mapping allows us to define the image $\phi^{*} \pi_{\sigma}^{\alpha}$ of the fractional Poisson measure $\pi_{\sigma}^{\alpha}$ under $\phi$ by $\phi^{*} \pi_{\sigma}^{\alpha}=\pi_{\sigma}^{\alpha} \circ \phi^{-1}$, more explicitly,

$$
\left(\phi^{*} \pi_{\sigma}^{\alpha}\right)(B):=\pi_{\sigma}^{\alpha}\left(\phi^{-1}(B)\right), \quad B \in \mathcal{B}(\Gamma) .
$$

The next proposition shows that the image measure $\phi^{*} \pi_{\sigma}^{\alpha}$ is again a fractional Poisson measure but with a different underlying measure.

Proposition 3.1. For any $\phi \in \operatorname{Diff}_{0}(M)$, the measure $\phi^{*} \pi_{\sigma}^{\alpha}$ is a fractional Poisson measure with intensity measure $\phi^{*} \sigma$, i.e.,

$$
\phi^{*} \pi_{\sigma}^{\alpha}=\pi_{\phi^{*} \sigma}^{\alpha} .
$$

Proof. It is sufficient to compute the Laplace transform of the measure $\phi^{*} \pi_{\sigma}^{\alpha}$. For any $f \in C_{0}(M)$

$$
\begin{aligned}
\int_{\Gamma} e^{\langle\gamma, f\rangle} d\left(\phi^{*} \pi_{\sigma}^{\alpha}\right)(\gamma) & =\int_{\Gamma} e^{\langle\phi(\gamma), f\rangle} d \pi_{\sigma}^{\alpha}(\gamma) \\
& =E_{\alpha}\left(\int_{\mathbb{R}^{d}}\left(e^{(f \circ \phi)(x)}-1\right) d \sigma(x)\right) \\
& =E_{\alpha}\left(\int_{\mathbb{R}^{d}}\left(e^{f(x)}-1\right) d\left(\phi^{*} \sigma\right)(x)\right) \\
& =\int_{\Gamma} e^{\langle\gamma, f\rangle} d \pi_{\phi^{*} \sigma}^{\alpha}(\gamma) .
\end{aligned}
$$

Theorem 3.1. The fractional Poisson measure $\pi_{\sigma}^{\alpha}$ is quasi-invariant with respect to the group $\operatorname{Diff}_{0}(M)$, and for any $\phi \in \operatorname{Diff}_{0}(M)$ the generalized Radon-Nikodym derivative is given by

$$
\Phi_{\sigma}^{\alpha}(\gamma):=\frac{d\left(\phi^{*} \pi_{\sigma}^{\alpha}\right)}{d \pi_{\sigma}}(\gamma)=\int_{0}^{\infty} \frac{d\left(\phi^{*} \pi_{\tau \sigma}\right)}{d \pi_{\sigma}}(\gamma) d \nu_{\alpha}(\tau) .
$$


Proof. Having in mind the previous proposition it is enough to show that

$$
\int_{\Gamma} e^{\langle\gamma, \varphi\rangle} \Phi_{\sigma}^{\alpha}(\gamma) d \pi_{\sigma}(\gamma)=\int_{\Gamma} e^{\langle\gamma, \varphi\rangle} d \pi_{\phi^{*} \sigma}^{\alpha}(\gamma), \quad \varphi \in \mathcal{D} .
$$

To show this we proceed as follows:

$$
\begin{aligned}
\int_{\Gamma} e^{\langle\gamma, \varphi\rangle} \Phi_{\sigma}^{\alpha}(\gamma) d \pi_{\sigma}(\gamma) & =\int_{\Gamma} e^{\langle\gamma, \varphi\rangle} \int_{0}^{\infty} \frac{d\left(\phi^{*} \pi_{\tau \sigma}\right)}{d \pi_{\sigma}}(\gamma) d \nu_{\alpha}(\tau) d \pi_{\sigma}(\gamma) \\
& =\int_{0}^{\infty} \int_{\Gamma} e^{\langle\gamma, \varphi\rangle} \frac{d\left(\phi^{*} \pi_{\tau \sigma}\right)}{d \pi_{\sigma}}(\gamma) d \pi_{\sigma}(\gamma) d \nu_{\alpha}(\tau) .
\end{aligned}
$$

The inner integral is solved as in Ref. 4 and is equal to

$$
\int_{\Gamma} e^{\langle\gamma, \varphi\rangle} \frac{d\left(\phi^{*} \pi_{\tau \sigma}\right)}{d \pi_{\sigma}}(\gamma) d \pi_{\sigma}(\gamma)=\int_{\Gamma} e^{\langle\gamma, \varphi\rangle} d \pi_{\tau \phi^{*} \sigma}(\gamma)
$$

Substituting we obtain

$$
\begin{aligned}
\int_{0}^{\infty} \int_{\Gamma} e^{\langle\gamma, \varphi\rangle} d \pi_{\tau \phi^{*} \sigma}(\gamma) d \nu_{\alpha}(\tau) & =\int_{\Gamma} e^{\langle\gamma, \varphi\rangle} \int_{0}^{\infty} d \pi_{\tau \phi^{*} \sigma}(\gamma) d \nu_{\alpha}(\tau) \\
& =\int_{\Gamma} e^{\langle\gamma, \varphi\rangle} d \pi_{\phi^{*} \sigma}^{\alpha}(\gamma)
\end{aligned}
$$

\section{Test and generalized functions on the fractional Lebesgue-Poisson space}

For $\alpha=1$, the description of the elements of the space $L^{2}\left(\Gamma, \pi_{\sigma}\right)$ of complex-valued square integrable functions by the corresponding chaos decomposition provides a unitary isomorphism between the spaces $L^{2}\left(\Gamma, \pi_{\sigma}\right)$ and $L^{2}\left(\Gamma_{0}, \lambda_{\sigma}^{1}\right)$. This unitary isomorphism is at the origin of the spaces of test and generalized functions on the Lebesgue-Poisson space $\left(\Gamma_{0}, \mathcal{B}\left(\Gamma_{0}\right), \lambda_{\sigma}^{1}\right)$ introduced in Ref. 7. For a generic $0<\alpha<$ 1 , it has been constructed in Ref. 3 a unitary isomorphism $I_{\alpha}$ between the spaces $L^{2}\left(\Gamma_{0}, \lambda_{\sigma}^{1}\right)$ and $L^{2}\left(\Gamma_{0}, \lambda_{\sigma}^{\alpha}\right)$,

$$
I_{\alpha}(G)=\frac{G}{\sqrt{E_{\alpha}^{(|\cdot|)}(0)}}, \quad G \in L^{2}\left(\Gamma_{0}, \lambda_{\sigma}^{1}\right) .
$$

As a result, the unitary isomorphism $I_{\alpha}$ leads to a natural construction of spaces of test and generalized functions on the fractional Lebesgue-Poisson space $\left(\Gamma_{0}, \mathcal{B}\left(\Gamma_{0}\right), \lambda_{\sigma}^{\alpha}\right)$. To carry out this construction, first we briefly recall the definition of the spaces of test and generalized functions for the case $\alpha=1$.

The starting point is the space $L_{\mathrm{Re}}^{2}(M, \sigma)$ of all real-valued square integrable functions with respect to the underlying measure $\sigma$ and a nuclear triple

$$
\operatorname{pr} \lim _{p \in \mathbb{N}} \mathcal{H}_{p}=\mathcal{N} \subset L_{R e}^{2}(M, \sigma) \subset \mathcal{N}^{\prime}=\operatorname{ind} \lim _{p \in \mathbb{N}} \mathcal{H}_{-p}
$$

such that

$$
\mathcal{D} \subset \mathcal{N} \subset L_{R e}^{2}(M, \sigma) \subset \mathcal{N}^{\prime} \subset \mathcal{D}^{\prime}
$$


More precisely, $\mathcal{N}$ is a nuclear space endowed with the projective limit topology of a family of Hilbert spaces $\mathcal{H}_{p}$ with the Hilbertian norm denote by $|\cdot|_{p}, p \in \mathbb{N}$, and $\mathcal{N}^{\prime}$ is the dual space of $\mathcal{N}$ with respect to $L_{R e}^{2}(M, \sigma)$ endowed with the inductive topology of the family of (Hilbert) dual spaces $\mathcal{H}_{-p}$ of $\mathcal{H}_{p}, p \in \mathbb{N}$, with respect to $L_{R e}^{2}(M, \sigma)$. We denote by $|\cdot|_{-p}$ the corresponding (Hilbertian) norm on the space $\mathcal{H}_{-p}, p \in \mathbb{N}$. Since there is no risk of confusion we preserve the notation $|\cdot|_{p}$ for the norm on the symmetric tensor power $\left(\mathcal{H}_{p, \mathbb{C}}\right)^{\hat{\otimes} n}$ of the complexified space $\mathcal{H}_{p, \mathbb{C}}$, and denoting by $\left(\mathcal{H}_{-p, \mathbb{C}}\right)^{\hat{\otimes} n}$ the corresponding dual space with respect to the space $\left(L^{2}(M, \sigma)\right)^{\hat{\otimes} n}$ of complex-valued functions, we also preserve the notation $|\cdot|_{-p}$ for the norm on $\left(\mathcal{H}_{-p, \mathbb{C}}\right)^{\hat{\otimes} n}, n \in \mathbb{N}$.

Within this framework, on the Lebesgue-Poisson space one defines a space of test functions $(\mathcal{N})_{1}^{1}$ by the projective limit of the Hilbert spaces

$$
\left(\mathcal{H}_{p}\right)_{q, 1}^{1}:=\left\{G \in L^{2}\left(\Gamma_{0}, \lambda_{\sigma}^{1}\right):\|G\|_{p, q, 1}<\infty\right\}, \quad p, q \in \mathbb{N},
$$

where, for each $G \in L^{2}\left(\Gamma_{0}, \lambda_{\sigma}^{1}\right)$,

$$
\|G\|_{p, q, 1}^{2}:=\sum_{n=0}^{\infty} 2^{n q}\left|G^{(n)}\right|_{p}^{2}, \quad G^{(n)}:=\left.G\right|_{\Gamma_{M}^{(n)}} .
$$

To introduce spaces of test and generalized functions in the fractional LebesguePoisson case, for each $0<\alpha<1$ fixed, let us consider the family of Hilbert spaces $\left(\mathcal{H}_{p}\right)_{q, \alpha}^{1}:=I_{\alpha}\left(\left(\mathcal{H}_{p}\right)_{q, 1}^{1}\right)$ with the natural Hilbertian norms given by

$$
\|G\|_{p, q, \alpha}^{2}:=\sum_{n=0}^{\infty} 2^{n q} E_{\alpha}^{(n)}(0)\left|G^{(n)}\right|_{p}^{2}=\sum_{n=0}^{\infty} \frac{2^{n q} n !}{\Gamma(\alpha n+1)}\left|G^{(n)}\right|_{p}^{2}, \quad p, q \in \mathbb{N} .
$$

This means, in particular,

$$
\left(\mathcal{H}_{p}\right)_{q, \alpha}^{1}=\left\{G \in L^{2}\left(\Gamma_{0}, \lambda_{\sigma}^{\alpha}\right):\|G\|_{p, q, \alpha}<\infty\right\}, \quad p, q \in \mathbb{N} .
$$

The space of test functions $(\mathcal{N})_{\alpha}^{1}$ is then defined as the projective limit of $\left(\mathcal{H}_{p}\right)_{q, \alpha}^{1}$, $p, q \in \mathbb{N}$. By the general duality theory the dual space $(\mathcal{N})_{\alpha}^{-1}$ of $(\mathcal{N})_{\alpha}^{1}$ (with respect to $\left.L^{2}\left(\Gamma_{0}, \lambda_{\sigma}^{\alpha}\right)\right)$ is given by the inductive limit of the dual spaces $\left(\mathcal{H}_{-p}\right)_{-q, \alpha}^{-1}$ of $\left(\mathcal{H}_{p}\right)_{q, \alpha}^{1}$ (with respect to $L^{2}\left(\Gamma_{0}, \lambda_{\sigma}^{\alpha}\right)$ ). In this way we have defined the nuclear triple

$$
(\mathcal{N})_{\alpha}^{1} \subset L^{2}\left(\Gamma_{0}, \lambda_{\sigma}^{\alpha}\right) \subset(\mathcal{N})_{\alpha}^{-1},
$$

where the (bilinear) dual pairing $\left\langle\langle\cdot, \cdot\rangle_{\alpha}\right.$ between $(\mathcal{N})_{\alpha}^{-1}$ and $(\mathcal{N})_{\alpha}^{1}$ is realized as an extension of the (sesquilinear) inner product on $L^{2}\left(\Gamma_{0}, \lambda_{\sigma}^{\alpha}\right)$ :

$$
\left\langle\left\langle G_{1}, G_{2}\right\rangle\right\rangle_{\alpha}=\left(G_{1}, \bar{G}_{2}\right)_{L^{2}\left(\Gamma_{0}, \lambda_{\sigma}^{\alpha}\right)}, \quad G_{1} \in(\mathcal{N})_{\alpha}^{1}, G_{2} \in L^{2}\left(\Gamma_{0}, \lambda_{\sigma}^{\alpha}\right)
$$

with $\bar{G}_{2}$ being the complex conjugate function of $G_{2}$.

Observe that as a direct consequence of this construction, for any $p, q \in \mathbb{N}$, $I_{\alpha}:\left(\mathcal{H}_{p}\right)_{q, 1}^{1} \rightarrow\left(\mathcal{H}_{p}\right)_{q, \alpha}^{1}$ is a unitary isomorphism. Therefore, the mapping $I_{\alpha}$ can be extended to the dual space $\left(\mathcal{H}_{-p}\right)_{-q, 1}^{-1}$ of $\left(\mathcal{H}_{p}\right)_{q, 1}^{1}$ (with respect to $L^{2}\left(\Gamma_{0}, \lambda_{\sigma}^{1}\right)$ ) and the extended mapping, also denoted by $I_{\alpha}$, maps $\left(\mathcal{H}_{-p}\right)_{-q, 1}^{-1}$ onto $\left(\mathcal{H}_{-p}\right)_{-q, \alpha}^{-1}$ as 
follows. For each $\Psi \in\left(\mathcal{H}_{-p}\right)_{-q, 1}^{-1}, I_{\alpha}(\Psi)$ is the unique element of $\left(\mathcal{H}_{-p}\right)_{-q, \alpha}^{-1}$ such that

$$
\left\langle\left\langle I_{\alpha}(\Psi), G\right\rangle\right\rangle_{\alpha}=\left\langle\left\langle\Psi, I_{\alpha}^{-1}(G)\right\rangle\right\rangle_{1}, \quad \forall G \in\left(\mathcal{H}_{p}\right)_{q, \alpha}^{1},
$$

where $\langle\langle\cdot, \cdot\rangle\rangle_{1}$ denotes the dual pairing between $(\mathcal{N})_{1}^{-1}$ and $(\mathcal{N})_{1}^{1}$. Moreover, for any $p, q \in \mathbb{N}, I_{\alpha}:\left(\mathcal{H}_{-p}\right)_{-q, 1}^{-1} \rightarrow\left(\mathcal{H}_{-p}\right)_{-q, \alpha}^{-1}$ is a unitary isomorphism. Hence, one may use these unitary isomorphims to transport the internal description of generalized functions on the Lebesgue-Poisson space to those on the fractional Lebesgue-Poisson space. As shown in Ref. 7, given the dual space $(\mathcal{N})_{1}^{-1}$ of the space $(\mathcal{N})_{1}^{1}$ of test functions (with respect to $L^{2}\left(\Gamma_{0}, \lambda_{\sigma}^{1}\right)$ ), for generalized functions $\Psi \in(\mathcal{N})_{1}^{-1}$ there is a natural decomposition $\left(\Psi^{(n)}\right)_{n=0}^{\infty}, \Psi^{(n)} \in \mathcal{D}_{\mathbb{C}}^{\prime \hat{\otimes} n}, n \in \mathbb{N}_{0}$, such that for all $G \in(\mathcal{N})_{1}^{1}$,

$$
\langle\langle\Psi, G\rangle\rangle_{1}=\sum_{n=0}^{\infty} \frac{1}{n !}\left\langle\Psi^{(n)}, G^{(n)}\right\rangle,
$$

and each Hilbert space $\left(\mathcal{H}_{-p}\right)_{-q, 1}^{-1}$ can be described as the set of elements $\Psi=$ $\left(\Psi^{(n)}\right)_{n=0}^{\infty} \in(\mathcal{N})_{1}^{-1}$ so that

$$
\|\Psi\|_{-p,-q, 1}^{2}=\sum_{n=0}^{\infty} \frac{2^{-n q}}{(n !)^{2}}\left|\Psi^{(n)}\right|_{-p}^{2}<\infty
$$

where $\|\cdot\|_{-p,-q, 1}$ denotes the Hilbertian norm on $\left(\mathcal{H}_{-p}\right)_{-q, 1}^{-1}$. Thus, in accordance with (5) and (6), for each $0<\alpha<1$ fixed, each generalized funtion $\Phi \in(\mathcal{N})_{\alpha}^{-1}$ has a decomposition of the form $\left(\Phi^{(n)}\right)_{n=0}^{\infty}, \Phi^{(n)} \in \mathcal{D}_{\mathbb{C}}^{\prime \hat{\otimes} n}, n \in \mathbb{N}_{0}$, such that for all $G \in(\mathcal{N})_{\alpha}^{1}$,

$$
\langle\langle\Phi, G\rangle\rangle_{\alpha}=\sum_{n=0}^{\infty} \frac{1}{\Gamma(\alpha n+1)}\left\langle\Phi^{(n)}, G^{(n)}\right\rangle,
$$

and each Hilbert space $\left(\mathcal{H}_{-p}\right)_{-q, \alpha}^{-1}, p, q \in \mathbb{N}$, can be described by

$$
\left(\mathcal{H}_{-p}\right)_{-q, \alpha}^{-1}=\left\{\Phi \in(\mathcal{N})_{\alpha}^{-1}:\|\Phi\|_{-p,-q, \alpha}<\infty\right\}
$$

with

$$
\|\Phi\|_{-p,-q, \alpha}^{2}=\sum_{n=0}^{\infty} \frac{2^{-n q}}{n ! \Gamma(\alpha n+1)}\left|\Phi^{(n)}\right|_{-p}^{2}, \quad \Phi=\left(\Phi^{(n)}\right)_{n=0}^{\infty} \in(\mathcal{N})_{\alpha}^{-1}
$$

\section{Acknowledgements}

We would like to express our gratitude for the splendid hospitality of our colleagues and friends M. Victoria Carpio-Bernido and Christopher C. Bernido during a very pleasant stay at Jagna during the 6th Jagna International Workshop. Financial support of the projects PEst-OE/MAT/UI0219/2011, PEst-OE/MAT/UI0209/2011 and PTDC/MAT/100983/2008 is gratefully acknowledged. 


\section{References}

1. N. Laskin, Commun. Nonlinear Sci. Numer. Simul. 8, 201 (2003), Chaotic transport and complexity in classical and quantum dynamics.

2. L. Beghin and E. Orsingher, Electronic Journal of Probability 14, 1790 (2009).

3. M. J. Oliveira, H. Ouerdiane, J. L. Silva and R. Vilela Mendes, The fractional Poisson measure in infinite dimensions (http://arxiv.org/abs/1002.2124, 2010).

4. S. Albeverio, Y. G. Kondratiev and M. Röckner, J. Funct. Anal. 154, 444 (1998).

5. W. Feller, An introduction to probability theory and its applications. Vol. II. Second edition, Second edition (John Wiley \& Sons Inc., New York, 1971).

6. Y. G. Kondratiev and T. Kuna, Infin. Dimens. Anal. Quantum Probab. Relat. Top. 5, 201 (2002).

7. Y. G. Kondratiev, T. Kuna and M. J. Oliveira, Methods Funct. Anal. Topology 8, 15 (2002). 